\title{
(Amino)cyclophosphazenes as multisite ligands for the synthesis of antitumoral and antibacterial silver(I) complexes
}

Elena Gascón ${ }^{[\mathrm{a}]}$, Sara Maisanaba ${ }^{[\mathrm{b}]}$, Isabel Otal ${ }^{[\mathrm{c}]}$, Eva Valero $^{[\mathrm{d}]}$, Guillermo Repetto ${ }^{[\mathrm{b}]}$, Peter G. Jones ${ }^{[\mathrm{e}]}$, Josefina Jiménez *[a]

[a] Dr. J. Jiménez, E. Gascón.

Departamento de Química Inorgánica, Facultad de Ciencias, Instituto de Síntesis Química y Catálisis Homogénea (ISQCH), Universidad de Zaragoza-CSIC, Pedro Cerbuna 12, 50009 Zaragoza, Spain. Email: jiimvil@unizar.es

${ }^{[b]}$ Dr. S. Maisanaba, Dr. G. Repetto.

Departamento de Biología Molecular e Ingeniería Bioquímica, Área de Toxicología, Universidad Pablo de Olavide, Ctra. Utrera, Km 1, 41013 Sevilla, Spain.

[c] Dr. I. Otal.

Grupo de Genética de Micobacterias, Departamento de Microbiología, Medicina Preventiva y Salud Pública, Universidad de Zaragoza, 50009 Zaragoza, Spain. Instituto de Salud Carlos III, CIBER de Enfermedades Respiratorias, E-28029 Madrid, Spain.

${ }^{[d]}$ Dr. E. Valero.

Departamento de Biología Molecular e Ingeniería Bioquímica, Área Nutrición y Bromatología, Universidad Pablo de Olavide, Ctra. Utrera, Km 1, 41013 Sevilla, Spain.

${ }^{\text {[e] }}$ Prof. P.G. Jones. Institut für Anorganische und Analytische Chemie, Technische Universität Braunschweig, Hagenring 30, D-38106, Braunschweig, Germany.

\section{SUPPORTING INFORMATION}

Table of Contents:

1. X-Ray Studies. Tables containing details of data collection and structure refinement for compounds 7 and 9 (Tables S1 and S2). Tables containing selected bond lengths and angles, and ring torsion angles for compounds 7 and 9 (Tables S3 and S4)

2. ${ }^{31} \mathrm{P}\left\{{ }^{1} \mathrm{H}\right\}$ and ${ }^{1} \mathrm{H}$ NMR spectra of compound $\mathbf{2}$ in DMSO measured over 48 hours, which is the time for the biological assays (Figures S1 and S2)

3. Cytotoxicity Studies. Table containing expected $\mathrm{IC}_{50}$ after $24 \mathrm{~h}$ to metallophosphazenes and their precursors exposure on MCF7 and HepG2 cells under microscope analysis (Table S5) 


\section{X-Ray Studies}

Table S1. Details of data collection and structure refinement for compound 7.2 $\mathrm{CH}_{2} \mathrm{Cl}_{2}$.

\begin{tabular}{|c|c|c|}
\hline formula & \multicolumn{2}{|c|}{$\mathrm{C}_{64} \mathrm{H}_{88} \mathrm{Ag}_{2} \mathrm{Cl}_{4} \mathrm{~F}_{6} \mathrm{~N}_{9} \mathrm{O}_{6} \mathrm{P}_{5} \mathrm{~S}_{2}$} \\
\hline crystal size [mm] & \multicolumn{2}{|c|}{$0.18 \times 0.18 \times 0.13$} \\
\hline Formula weight & \multicolumn{2}{|l|}{1769.94} \\
\hline Wavelength & \multicolumn{2}{|l|}{$0.71073 \AA$} \\
\hline crystal system & \multicolumn{2}{|l|}{ Triclinic } \\
\hline space group & \multicolumn{2}{|l|}{$P(-1)$} \\
\hline \multirow[t]{3}{*}{ unit cell dimensions } & $a=13.367(3) \AA$ & $\alpha=84.862(6)^{\circ}$ \\
\hline & $\mathrm{b}=15.117(3) \AA$ & $\beta=74.356(6)^{\circ}$ \\
\hline & $C=21.521(4) \AA$ & $\gamma=67.845(6)^{\circ}$ \\
\hline volume $\left[\AA^{3}\right]$ & \multicolumn{2}{|l|}{$3878.1(14)$} \\
\hline$Z$ & \\
\hline density (calculated) $\left[\mathrm{g} \mathrm{cm}^{-3}\right]$ & 1.516 & \\
\hline absorption coefficient $\left[\mathrm{mm}^{-1}\right]$ & \multicolumn{2}{|l|}{0.867} \\
\hline$F(000)$ & \multicolumn{2}{|l|}{1812} \\
\hline Temperature & \multirow{2}{*}{\multicolumn{2}{|c|}{$\begin{array}{l}143(2) \mathrm{K} \\
145 \text { to } 26.37\end{array}$}} \\
\hline theta range for data collection $\left[{ }^{\circ}\right]$ & & \\
\hline index ranges & \multicolumn{2}{|c|}{$-16<=h<=16,-18<=k<=18,-26<=\mid<=26$} \\
\hline reflections collected & \multicolumn{2}{|c|}{49187} \\
\hline independent reflections & \multicolumn{2}{|c|}{$15826[R($ int $)=0.0726]$} \\
\hline completeness to theta $=26.00^{\circ}$ & \multicolumn{2}{|c|}{$99.8 \%$} \\
\hline absorption correction & \multicolumn{2}{|c|}{ Semi-empirical from equivalents } \\
\hline Max. and min. transmission & \multicolumn{2}{|c|}{0.928 and 0.776} \\
\hline $\begin{array}{l}\text { refinement method } \\
\text { data / restraints / parameters }\end{array}$ & \multicolumn{2}{|c|}{$\begin{array}{l}\text { Full-matrix least-squares on } \mathrm{F}^{2} \\
15826 \text { / } 429 \text { / } 838\end{array}$} \\
\hline $\begin{array}{l}\text { Goodness-of-fit on } F^{2} \\
\text { final } R \text { indices }[l>2 \text { sigma }(\mathrm{I})] \\
R \text { indices (all data) } \\
\text { largest diff. peak and hole }\left[\mathrm{e} \AA^{-3}\right]\end{array}$ & \multicolumn{2}{|c|}{$\begin{array}{l}0.93 \\
R 1=0.0443, w R 2=0.1029 \\
R 1=0.0777, w R 2=0.1160 \\
0.97 \text { and }-0.92\end{array}$} \\
\hline
\end{tabular}


Table S2. Details of data collection and structure refinement for compound 9.1/2 $\mathrm{C}_{6} \mathrm{H}_{14}$

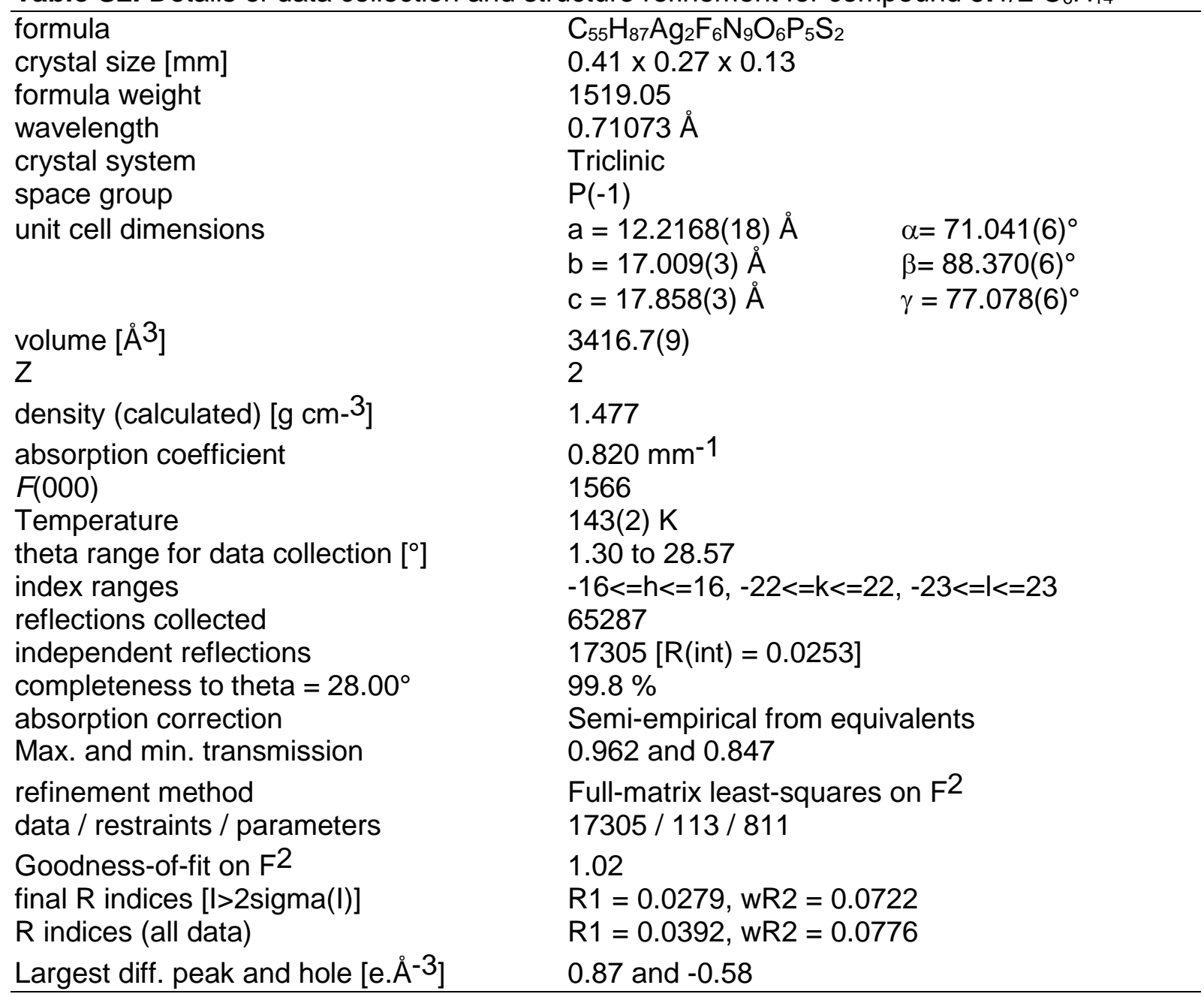


Table S3. Selected bond lengths $[\AA]$ and angles $\left[{ }^{\circ}\right]$, and ring torsion angles $\left[{ }^{\circ}\right]$ for compound $7.2 \mathrm{CH}_{2} \mathrm{Cl}_{2}$.

\begin{tabular}{lllr}
\hline $\mathrm{Ag}(1)-\mathrm{N}(1)$ & $2.123(3)$ & $\mathrm{N}(2)-\mathrm{P}(2)-\mathrm{N}(1)$ & $112.26(15)$ \\
$\mathrm{Ag}(1)-\mathrm{P}(4)$ & $2.3371(10)$ & $\mathrm{N}(6)-\mathrm{P}(2)-\mathrm{N}(1)$ & $103.03(15)$ \\
$\mathrm{Ag}(2)-\mathrm{N}(3)$ & $2.162(3)$ & $\mathrm{N}(2)-\mathrm{P}(2)-\mathrm{N}(7)$ & $108.07(15)$ \\
$\mathrm{Ag}(2)-\mathrm{P}(5)$ & $2.3380(11)$ & $\mathrm{N}(6)-\mathrm{P}(2)-\mathrm{N}(7)$ & $102.75(15)$ \\
$\mathrm{P}(1)-\mathrm{N}(3)$ & $1.613(3)$ & $\mathrm{N}(1)-\mathrm{P}(2)-\mathrm{N}(7)$ & $113.69(16)$ \\
$\mathrm{P}(1)-\mathrm{N}(1)$ & $1.622(3)$ & $\mathrm{N}(2)-\mathrm{P}(3)-\mathrm{N}(3)$ & $112.78(15)$ \\
$\mathrm{P}(1)-\mathrm{N}(4)$ & $1.625(3)$ & $\mathrm{N}(2)-\mathrm{P}(3)-\mathrm{N}(8)$ & $117.29(17)$ \\
$\mathrm{P}(1)-\mathrm{N}(5)$ & $1.652(3)$ & $\mathrm{N}(3)-\mathrm{P}(3)-\mathrm{N}(8)$ & $103.08(17)$ \\
$\mathrm{P}(2)-\mathrm{N}(2)$ & $1.585(3)$ & $\mathrm{N}(2)-\mathrm{P}(3)-\mathrm{N}(9)$ & $106.85(17)$ \\
$\mathrm{P}(2)-\mathrm{N}(6)$ & $1.628(3)$ & $\mathrm{N}(3)-\mathrm{P}(3)-\mathrm{N}(9)$ & $114.31(16)$ \\
$\mathrm{P}(2)-\mathrm{N}(1)$ & $1.639(3)$ & $\mathrm{N}(8)-\mathrm{P}(3)-\mathrm{N}(9)$ & $102.32(17)$ \\
$\mathrm{P}(2)-\mathrm{N}(7)$ & $1.642(3)$ & $\mathrm{P}(1)-\mathrm{N}(1)-\mathrm{P}(2)$ & $124.60(17)$ \\
$\mathrm{P}(3)-\mathrm{N}(2)$ & $1.580(3)$ & $\mathrm{P}(1)-\mathrm{N}(1)-\mathrm{Ag}(1)$ & $116.74(15)$ \\
$\mathrm{P}(3)-\mathrm{N}(3)$ & $1.634(3)$ & $\mathrm{P}(2)-\mathrm{N}(1)-\mathrm{Ag}(1)$ & $118.46(15)$ \\
$\mathrm{P}(3)-\mathrm{N}(8)$ & $1.643(3)$ & $\mathrm{P}(3)-\mathrm{N}(2)-\mathrm{P}(2)$ & $126.75(19)$ \\
$\mathrm{P}(3)-\mathrm{N}(9)$ & $1.652(3)$ & $\mathrm{P}(1)-\mathrm{N}(3)-\mathrm{P}(3)$ & $124.97(19)$ \\
$\mathrm{N}(1)-\mathrm{Ag}(1)-\mathrm{P}(4)$ & $167.97(8)$ & $\mathrm{P}(1)-\mathrm{N}(3)-\mathrm{Ag}(2)$ & $121.52(16)$ \\
$\mathrm{N}(3)-\mathrm{Ag}(2)-\mathrm{P}(5)$ & $165.57(8)$ & $\mathrm{P}(3)-\mathrm{N}(3)-\mathrm{Ag}(2)$ & $113.28(14)$ \\
$\mathrm{N}(3)-\mathrm{P}(1)-\mathrm{N}(1)$ & $112.08(15)$ & $\mathrm{N}(3)-\mathrm{P}(1)-\mathrm{N}(1)-\mathrm{P}(2)$ & $9.9(3)$ \\
$\mathrm{N}(3)-\mathrm{P}(1)-\mathrm{N}(4)$ & $104.07(15)$ & $\mathrm{N}(2)-\mathrm{P}(2)-\mathrm{N}(1)-\mathrm{P}(1)$ & $-24.5(3)$ \\
$\mathrm{N}(1)-\mathrm{P}(1)-\mathrm{N}(4)$ & $116.94(16)$ & $\mathrm{N}(3)-\mathrm{P}(3)-\mathrm{N}(2)-\mathrm{P}(2)$ & $4.9(3)$ \\
$\mathrm{N}(3)-\mathrm{P}(1)-\mathrm{N}(5)$ & $116.44(15)$ & $\mathrm{N}(1)-\mathrm{P}(2)-\mathrm{N}(2)-\mathrm{P}(3)$ & $16.3(3)$ \\
$\mathrm{N}(1)-\mathrm{P}(1)-\mathrm{N}(5)$ & $104.12(15)$ & $\mathrm{N}(1)-\mathrm{P}(1)-\mathrm{N}(3)-\mathrm{P}(3)$ & $15.3(3)$ \\
$\mathrm{N}(4)-\mathrm{P}(1)-\mathrm{N}(5)$ & $103.36(16)$ & $\mathrm{N}(2)-\mathrm{P}(3)-\mathrm{N}(3)-\mathrm{P}(1)$ & $-22.5(3)$ \\
$\mathrm{N}(2)-\mathrm{P}(2)-\mathrm{N}(6)$ & $116.82(16)$ & & \\
\hline $\mathrm{S}(6)$ & & &
\end{tabular}

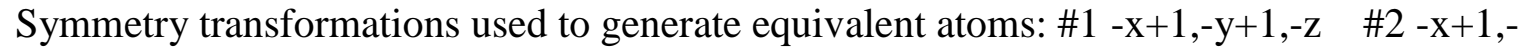
$\mathrm{y}+1,-\mathrm{z}+1$ 
Table S4. Selected bond lengths $[\AA]$ and angles $\left[{ }^{\circ}\right]$, and ring torsion angles $\left[{ }^{\circ}\right]$ for compound 9.1/2 $\mathrm{C}_{6} \mathrm{H}_{14}$

\begin{tabular}{llll}
\hline $\mathrm{Ag}(1)-\mathrm{N}(1)$ & $2.1507(14)$ & $\mathrm{N}(2)-\mathrm{P}(2)-\mathrm{N}(1)$ & $112.57(8)$ \\
$\mathrm{Ag}(1)-\mathrm{P}(4)$ & $2.3498(5)$ & $\mathrm{N}(6)-\mathrm{P}(2)-\mathrm{N}(1)$ & $102.06(8)$ \\
$\mathrm{Ag}(2)-\mathrm{N}(3)$ & $2.1463(14)$ & $\mathrm{N}(2)-\mathrm{P}(2)-\mathrm{N}(7)$ & $105.77(8)$ \\
$\mathrm{Ag}(2)-\mathrm{P}(5)$ & $2.3474(6)$ & $\mathrm{N}(6)-\mathrm{P}(2)-\mathrm{N}(7)$ & $102.69(8)$ \\
$\mathrm{P}(1)-\mathrm{N}(3)$ & $1.6168(15)$ & $\mathrm{N}(1)-\mathrm{P}(2)-\mathrm{N}(7)$ & $115.33(8)$ \\
$\mathrm{P}(1)-\mathrm{N}(1)$ & $1.6173(15)$ & $\mathrm{N}(2)-\mathrm{P}(3)-\mathrm{N}(3)$ & $113.40(8)$ \\
$\mathrm{P}(1)-\mathrm{N}(4)$ & $1.6258(17)$ & $\mathrm{N}(2)-\mathrm{P}(3)-\mathrm{N}(8)$ & $116.03(8)$ \\
$\mathrm{P}(1)-\mathrm{N}(5)$ & $1.6529(16)$ & $\mathrm{N}(3)-\mathrm{P}(3)-\mathrm{N}(8)$ & $102.92(8)$ \\
$\mathrm{P}(2)-\mathrm{N}(2)$ & $1.5878(15)$ & $\mathrm{N}(2)-\mathrm{P}(3)-\mathrm{N}(9)$ & $107.10(8)$ \\
$\mathrm{P}(2)-\mathrm{N}(6)$ & $1.6362(16)$ & $\mathrm{N}(3)-\mathrm{P}(3)-\mathrm{N}(9)$ & $113.51(8)$ \\
$\mathrm{P}(2)-\mathrm{N}(1)$ & $1.6396(15)$ & $\mathrm{N}(8)-\mathrm{P}(3)-\mathrm{N}(9)$ & $103.64(8)$ \\
$\mathrm{P}(2)-\mathrm{N}(7)$ & $1.6501(16)$ & $\mathrm{P}(1)-\mathrm{N}(1)-\mathrm{P}(2)$ & $122.56(9)$ \\
$\mathrm{P}(3)-\mathrm{N}(2)$ & $1.5843(15)$ & $\mathrm{P}(1)-\mathrm{N}(1)-\mathrm{Ag}(1)$ & $120.07(8)$ \\
$\mathrm{P}(3)-\mathrm{N}(3)$ & $1.6342(15)$ & $\mathrm{P}(2)-\mathrm{N}(1)-\mathrm{Ag}(1)$ & $116.23(8)$ \\
$\mathrm{P}(3)-\mathrm{N}(8)$ & $1.6403(16)$ & $\mathrm{P}(3)-\mathrm{N}(2)-\mathrm{P}(2)$ & $126.64(10)$ \\
$\mathrm{P}(3)-\mathrm{N}(9)$ & $1.6561(16)$ & $\mathrm{P}(1)-\mathrm{N}(3)-\mathrm{P}(3)$ & $124.32(9)$ \\
$\mathrm{N}(1)-\mathrm{Ag}(1)-\mathrm{P}(4)$ & $168.26(4)$ & $\mathrm{P}(1)-\mathrm{N}(3)-\mathrm{Ag}(2)$ & $120.17(8)$ \\
$\mathrm{N}(3)-\mathrm{Ag}(2)-\mathrm{P}(5)$ & $177.26(4)$ & $\mathrm{P}(3)-\mathrm{N}(3)-\mathrm{Ag}(2)$ & $115.49(7)$ \\
$\mathrm{N}(3)-\mathrm{P}(1)-\mathrm{N}(1)$ & $112.09(8)$ & $\mathrm{N}(3)-\mathrm{P}(1)-\mathrm{N}(1)-\mathrm{P}(2)$ & $32.87(13)$ \\
$\mathrm{N}(3)-\mathrm{P}(1)-\mathrm{N}(4)$ & $106.41(8)$ & $\mathrm{N}(2)-\mathrm{P}(2)-\mathrm{N}(1)-\mathrm{P}(1)$ & $-25.45(14)$ \\
$\mathrm{N}(1)-\mathrm{P}(1)-\mathrm{N}(4)$ & $115.88(9)$ & $\mathrm{N}(3)-\mathrm{P}(3)-\mathrm{N}(2)-\mathrm{P}(2)$ & $16.91(15)$ \\
$\mathrm{N}(3)-\mathrm{P}(1)-\mathrm{N}(5)$ & $112.88(8)$ & $\mathrm{N}(1)-\mathrm{P}(2)-\mathrm{N}(2)-\mathrm{P}(3)$ & $-1.58(15)$ \\
$\mathrm{N}(1)-\mathrm{P}(1)-\mathrm{N}(5)$ & $104.61(8)$ & $\mathrm{N}(1)-\mathrm{P}(1)-\mathrm{N}(3)-\mathrm{P}(3)$ & $-15.12(14)$ \\
$\mathrm{N}(4)-\mathrm{P}(1)-\mathrm{N}(5)$ & $104.90(9)$ & $\mathrm{N}(2)-\mathrm{P}(3)-\mathrm{N}(3)-\mathrm{P}(1)$ & $-7.64(14)$ \\
$\mathrm{N}(2)-\mathrm{P}(2)-\mathrm{N}(6)$ & $118.45(8)$ & &
\end{tabular}

Symmetry transformations used to generate equivalent atoms: \#1 -x,-y+1,-z+2 
2. ${ }^{31} \mathrm{P}\left\{{ }^{1} \mathrm{H}\right\}$ and ${ }^{1} \mathrm{H}$ NMR spectra of compound 2 in DMSO measured over 48 hours
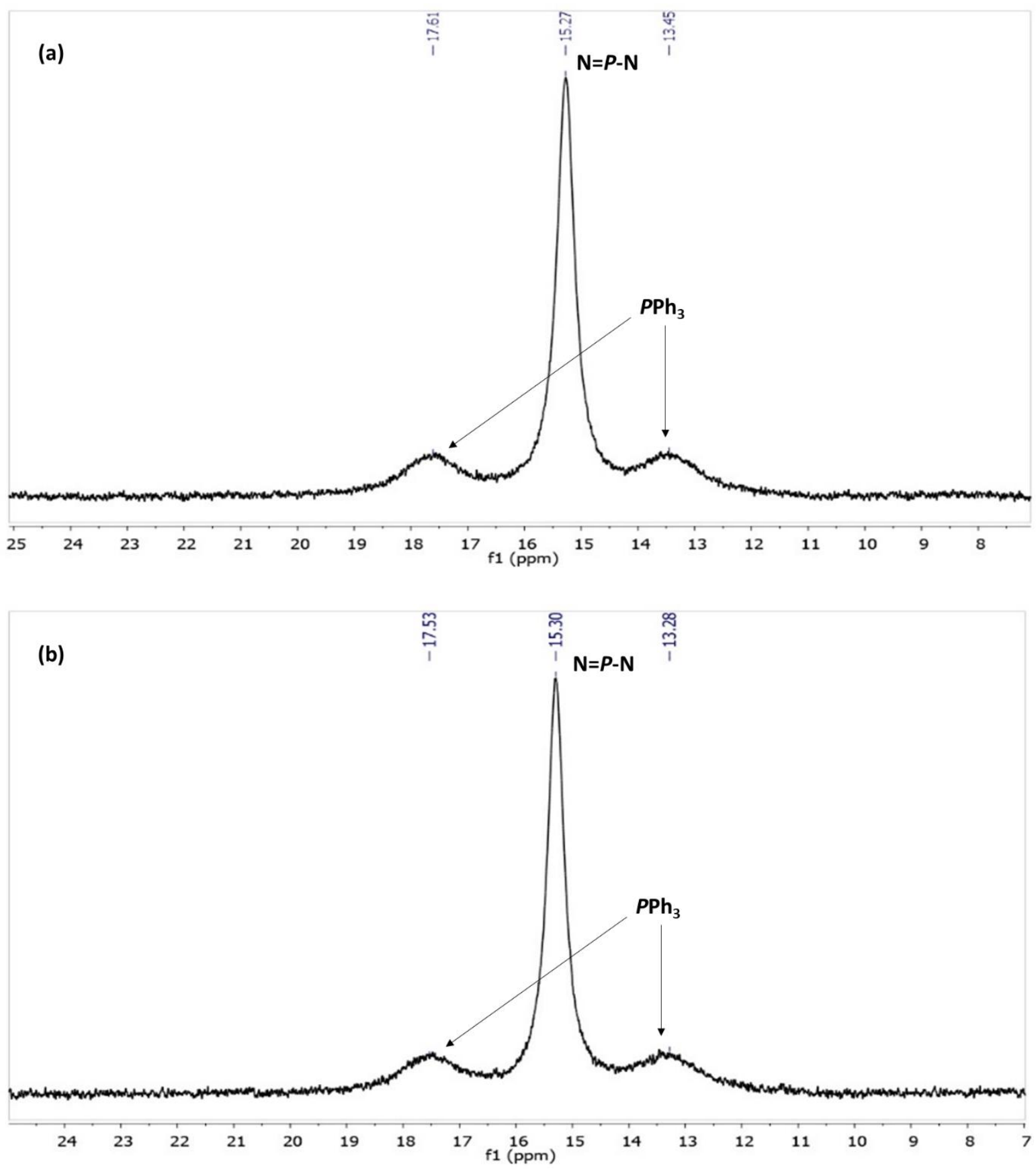

Figure S1. (a) ${ }^{31} \mathrm{P}\left\{{ }^{1} \mathrm{H}\right\}$ NMR spectra of compound 2 measured in DMSO at RT and (b) after 48 hours dissolved in DMSO. 
(a)

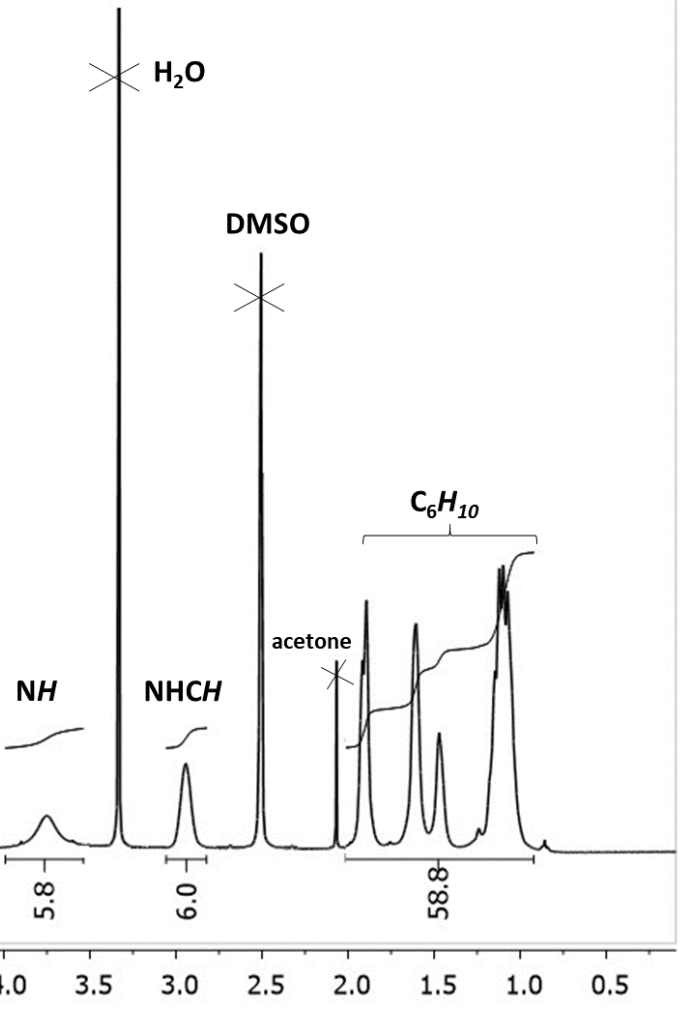

(b)

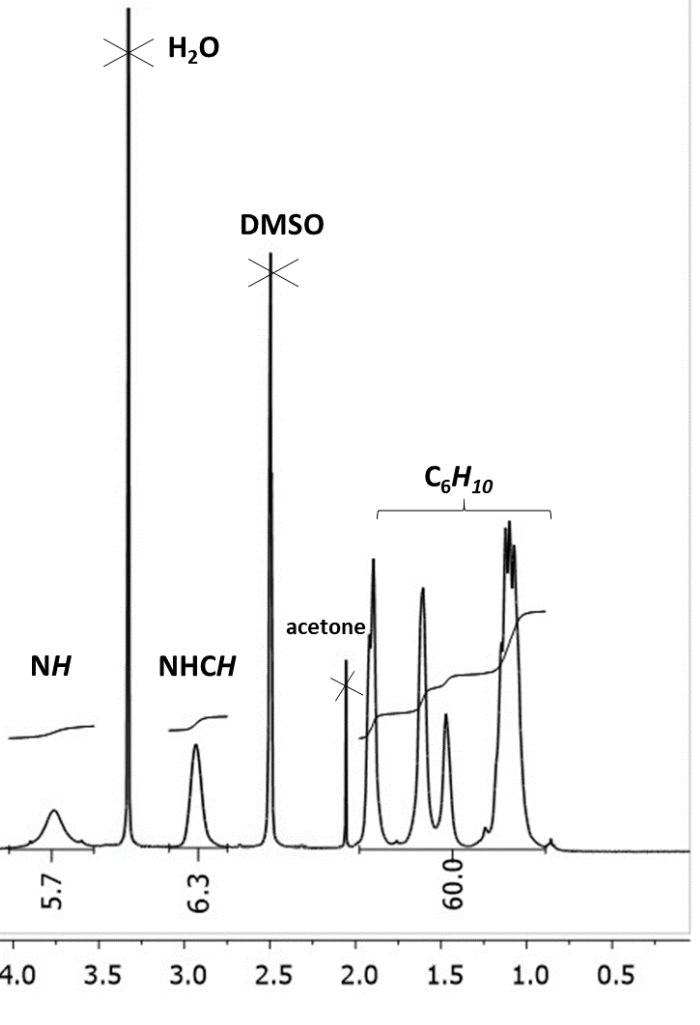

Figure S2. (a) ${ }^{1} \mathrm{H}$ NMR spectra of compound 2 measured in DMSO at RT and (b) after 48 hours dissolved in DMSO. 


\section{Cytotoxicity studies}

Table S5. Expected $I_{50}$ after $24 \mathrm{~h}$ to metallophosphazenes and their precursors exposure on MCF7 and HepG2 cells under microscope analysis.

\begin{tabular}{|c|c|c|}
\hline \multirow{2}{*}{ Compound } & \multicolumn{2}{|c|}{ Expected Range IC50 at $24 \mathrm{~h}(\mu \mathrm{M})$} \\
\hline & MCF7 & HepG2 \\
\hline $\mathrm{N}_{3} \mathrm{P}_{3}(\mathrm{NHCy})_{6}$ (phos-1) & $>25$ & $>25$ \\
\hline$\left[\mathrm{N}_{3} \mathrm{P}_{3}(\mathrm{NHCy})_{6}\left\{\mathrm{Ag}\left(\mathrm{PPh}_{3}\right)\right\}_{2}\right](\mathrm{TfO})_{2}(\mathbf{2})$ & $2-4$ & $1-2$ \\
\hline$\left[\mathrm{N}_{3} \mathrm{P}_{3}(\mathrm{NHCy})_{6}\left\{\mathrm{Ag}\left(\mathrm{PPh}_{3}\right)\right\}_{3}\right](\mathrm{TfO})_{3}(\mathbf{3})$ & $1-2$ & $0.5-1$ \\
\hline$\left[\mathrm{Ag}(\mathrm{OTf}) \mathrm{PPh}_{3}\right]$ & $5-10$ & $5-10$ \\
\hline$\left[\mathrm{N}_{3} \mathrm{P}_{3}(\mathrm{NHCy})_{6}\left\{\mathrm{Ag}\left(\mathrm{PPh}_{2} \mathrm{Me}\right)\right\}_{2}\right](\mathrm{TfO})_{2}(\mathbf{4})$ & $4-6$ & $2-4$ \\
\hline$\left[\mathrm{N}_{3} \mathrm{P}_{3}(\mathrm{NHCy})_{6}\left\{\mathrm{Ag}\left(\mathrm{PPh} \mathrm{Me}_{2}\right\}_{3}\right](\mathrm{TfO})_{3}(\mathbf{5})\right.$ & $2-4$ & $1-2$ \\
\hline$\left[\mathrm{Ag}(\mathrm{OTf}) \mathrm{PPh}_{2} \mathrm{Me}\right]$ & $10-20$ & $10-20$ \\
\hline$\left[\mathrm{N}_{3} \mathrm{P}_{3}(\mathrm{NHCy})_{6}\{\mathrm{Ag}(\mathrm{TPA})\}_{3}\right](\mathrm{TfO})_{3}(\mathbf{6})$ & $6-8$ & $4-6$ \\
\hline$[\mathrm{Ag}(\mathrm{OTf}) \mathrm{TPA}]$ & $20-40$ & $20-40$ \\
\hline $\mathrm{N}_{3} \mathrm{P}_{3}\left(\mathrm{NMe}_{2}\right)_{3}(\mathrm{NHCy})_{3}$ (phos-3) & $20-40$ & $10-20$ \\
\hline $\left.\mathrm{N}_{3} \mathrm{P}_{3}\left(\mathrm{NMe}_{2}\right)_{3}(\mathrm{NHCy})_{3}\left\{\mathrm{Ag}\left(\mathrm{PPh}_{2} \mathrm{Me}\right)\right\}_{2}\right](\mathrm{TfO})_{2}(\mathbf{9})$ & $4-6$ & $2-4$ \\
\hline $\left.\mathrm{N}_{3} \mathrm{P}_{3}\left(\mathrm{NMe}_{2}\right)_{3}(\mathrm{NHCy})_{3}\left\{\mathrm{Ag}\left(\mathrm{PPh}_{2} \mathrm{Me}\right)\right\}_{3}\right](\mathrm{TfO})_{3}(\mathbf{1 0})$ & $4-6$ & $1-2$ \\
\hline
\end{tabular}

